\title{
Renormalization of effective interactions in a negative charge transfer insulator
}

\author{
Priyanka Seth, ${ }^{1,2}$ Oleg E. Peil,${ }^{1,3,4,{ }^{*}}$ Leonid Pourovskii, ${ }^{1,4}$ Markus Betzinger, ${ }^{5}$ Christoph Friedrich, ${ }^{5}$ Olivier Parcollet, ${ }^{2}$ \\ Silke Biermann, ${ }^{1}$ Ferdi Aryasetiawan, ${ }^{6}$ and Antoine Georges ${ }^{1,3,4}$ \\ ${ }^{1}$ Centre de Physique Théorique, École Polytechnique, CNRS, Université Paris-Saclay, 91128 Palaiseau, France \\ ${ }^{2}$ Institut de Physique Théorique (IPhT), CEA, CNRS, 91191 Gif-sur-Yvette, France \\ ${ }^{3}$ Department of Quantum Matter Physics, University of Geneva, 24 Quai Ernest-Ansermet, 1211 Geneva 4, Switzerland \\ ${ }^{4}$ Collège de France, 11 place Marcelin Berthelot, 75005 Paris, France \\ ${ }^{5}$ Peter Grünberg Institut and Institute for Advanced Simulation, Forschungszentrum Jülich and JARA, 52425 Jülich, Germany \\ ${ }^{6}$ Department of Physics, Division of Mathematical Physics, Professorsgatan 1, 22362 Lund, Sweden \\ (Received 3 August 2017; revised manuscript received 13 November 2017; published 21 November 2017)
}

\begin{abstract}
We compute from first principles the effective interaction parameters appropriate for a low-energy description of the rare-earth nickelate $\mathrm{LuNiO}_{3}$ involving the partially occupied $e_{g}$ states only. The calculation uses the constrained random-phase approximation and reveals that the effective on-site Coulomb repulsion is strongly reduced by screening effects involving the oxygen- $p$ and nickel- $t_{2 g}$ states. The long-range component of the effective low-energy interaction is also found to be sizable. As a result, the effective on-site interaction between parallel-spin electrons is reduced down to a small negative value. This validates effective low-energy theories of these materials that were proposed earlier. Electronic structure methods combined with dynamical mean-field theory are used to construct and solve an appropriate low-energy model and explore its phase diagram as a function of the on-site repulsion and Hund's coupling. For the calculated values of these effective interactions, we find that in agreement with experiments, $\mathrm{LuNiO}_{3}$ is a metal without disproportionation of the $e_{g}$ occupancy when considered in its orthorhombic structure, while the monoclinic phase is a disproportionated insulator.
\end{abstract}

DOI: 10.1103/PhysRevB.96.205139

\section{INTRODUCTION}

The interplay between the atomic physics and strong covalent bonding in transition-metal oxides (TMOs) results in a variety of fascinating phenomena [1]. The energy scale spanned by the hybridized states formed by the $d$ orbitals of the transition metal and the $p$ states of oxygen is typically of the order of $10 \mathrm{eV}$. However, it is often useful for a physical understanding to use a "low-energy" description in which only a subset of the metal-oxygen antibonding states is retained, namely, the partially occupied states in proximity to the Fermi level. Those usually span a narrower energy window of a few electron volts. Moving from the full high-energy description to a low-energy model allows one to reduce the dimension of the Hilbert space considerably, and quite often provides physical insight into the behavior of a material. This is particularly relevant to late transition-metal oxides involving antibonding $e_{g}$ orbitals, as exemplified by the Zhang-Rice single-band picture of cuprates [2].

A price to pay for this simplification is the renormalization of interaction parameters when high-energy states are integrated out. These renormalizations can be large, and evaluating the proper values of low-energy interactions is a challenging problem of great practical importance. In all TMOs, an important interaction is the Coulomb repulsion $U_{d d}$ between localized, atomiclike $d$ states of the TM cation. In late TMOs, however, the energy scale relevant for low-energy states is the charge transfer energy, which can be much smaller [3] than $U_{d d}$.

A class of materials in which this issue is particularly relevant is the family of rare-earth nickelates, $R \mathrm{NiO}_{3}$. These materials have a very large degree of covalency between the $\mathrm{Ni}$

\footnotetext{
*Corresponding author: Oleg.Peil@gmail.com
}

and $\mathrm{O}$ states [4]. This may result in the charge transfer energy being very small in magnitude and possibly negative [5-9], leading to the appearance of holes on ligand (oxygen) states in the ground state [10]. A direct confirmation of the presence of ligand holes has been recently provided by x-ray absorption and resonant inelastic $\mathrm{x}$-ray scattering experiments [11].

The metal-insulator transition (MIT) of the $R \mathrm{NiO}_{3}$ series is accompanied by a structural transition from the high- $T$ orthorhombic structure to a low- $T$ monoclinic structure. In the latter, the uniform octahedra of the orthorhombic structure distort into a set of compressed octahedra with short $\mathrm{Ni}-\mathrm{O}$ bonds (SBs) and a set of expanded octahedra with long bonds (LBs). A qualitative, somewhat extreme picture of the low- $T$ phase $[8,12]$ is to assign the configuration $d^{8}$ to the Ni sites of the LB octahedra and $d^{8} \bar{L}^{2}$ (with two ligand holes delocalized on neighboring oxygens) to the SB octahedra. This is in contrast to the nominal valence $\mathrm{Ni}-d^{7}$ suggested by a naive counting in the ionic limit (with $R^{3+}, \mathrm{O}^{2-}$ ) so that the picture above can be summarized as $d^{7}+d^{7} \rightarrow d^{8}+d^{8} \bar{L}^{2}$. Correspondingly, in this extreme picture, the LB sites would carry a spin-one magnetic moment, while the SB sites would carry no magnetic moment (the Ni moment being screened by the oxygen holes [12]). Note that this disproportionation does not necessarily correspond to a large-amplitude charge ordering since each oxygen actually belongs to both a SB and LB octahedron so that the average charge on each octahedron can remain weakly modulated or even uniform.

Subedi et al. [13] recently proposed a low-energy description of the electronic structure of the $R \mathrm{NiO}_{3}$ series, involving only the $p-d$ hybridized antibonding states with $e_{g}$ symmetry close to the Fermi level. In this description, the above disproportionation can be viewed as $e_{g}^{1}+e_{g}^{1} \rightarrow e_{g}^{2}+e_{g}^{0}$ (Fig. 1). Building on earlier ideas by Mazin et al. [14], these authors showed that such a disproportionation is favored by a 


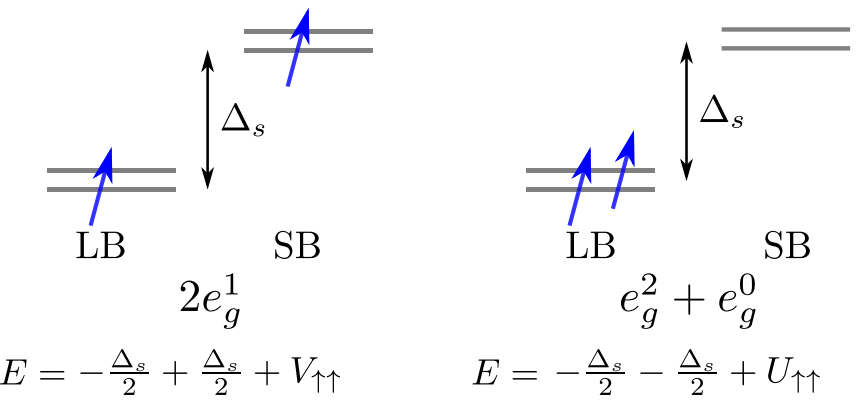

FIG. 1. Schematic picture of the disproportionation associated with the metal-insulator transition for a two-site model consisting of a LB site and a SB site. The energy of the LB site is lowered, compared to that of the SB site, by the Peierls energy $\Delta_{s}$. Each site carries two $e_{g}$ orbitals. In the doubly occupied configuration, each orbital is occupied by a single electron with parallel spins, in accordance with Hund's rules. An on-site interaction $U^{\sigma \sigma}$ between electrons with parallel spins is considered, as well as an intersite interaction $V^{\sigma \sigma}$. In the atomic limit where hopping is neglected, the criterion for stabilizing the disproportionated state $e_{g}^{2}+e_{g}^{0}$ over the uniform one $e_{g}^{1}+e_{g}^{1}$ reads $U^{\sigma \sigma}-V^{\sigma \sigma}<\Delta_{s}$. The extension of this criterion to a whole lattice in the presence of hopping is discussed in the main text.

strong reduction of the effective $U$ acting on the low-energy $e_{g}$ states and by a large value of the low-energy Hund's coupling $J$. More precisely, the monoclinic distortion splits the low-energy $e_{g}$ states into two groups of states separated by a Peierls-like energy gap $\Delta_{s}$ (note that this gap opens at the energy corresponding to half filling, and is hence not directly responsible for the transition into the insulating state of these nominally quarter-filled compounds). Using dynamical mean-field theory (DMFT) [15] in combination with density functional calculations, a $U-J$ phase diagram was established for the low-energy model, demonstrating that a disproportionated insulating phase is present in the range of coupling parameters where the parallel-spin interaction $U^{\sigma \sigma}=U-3 J$ is smaller than the Peierls gap $\Delta_{s}$.

The low-energy picture of Subedi et al. [13] is in good agreement both with experiments (e.g., optical spectroscopy $[16,17])$ and with earlier DMFT calculations, including all Ni- $d$ and O- $p$ states [12]. However, the question of whether the strongly renormalized value of the effective low-energy interaction $U^{\sigma \sigma}=U-3 J$ is indeed realistic remains widely open. A first-principles calculation of these low-energy effective interactions is obviously highly desirable. Furthermore, Ref. [13] did not consider the role of intersite interactions, which are surely induced when downfolding onto a low-energy model and are known to be important in materials with electronic disproportionation or charge ordering [18-26]. When the intersite interaction $V$ between the LB and SB sites is included, the more accurate condition for charge disproportionation becomes $U_{\sigma \sigma}-V_{\sigma \sigma}<\Delta_{s}$ for the two-site case treated in the atomic limit, as depicted in Fig. 1. For the full problem, one needs to include hopping, correctly treat the lattice connectivity, and also include the effect of longrange Coulomb interactions, which are important in insulators. There is little experimental spectroscopic information on such long-range interactions. The aim of the present paper is to attempt a first-principles determination of the appropriate lowenergy parameters, and examine the physical consequences of the obtained values in light of the issues discussed above.

The approach that we shall adopt is the constrained randomphase approximation (cRPA) [27]. This method has proven successful in calculating interaction parameters between electrons in localized $d$ or $f$ states assumed to be screened by more extended $s$ and $p$ states [28-34]. In this paper, we apply this method to calculate the interaction parameters corresponding to low-energy states of $\mathrm{LuNiO}_{3}$, which exhibits the largest distortion amongst the family of $R \mathrm{NiO}_{3}$. It is worth emphasizing that such a system represents a true challenge to cRPA because the contributions to screening come both from extended O- $p$, with possible ligand holes, which are very close in energy to the $e_{g}$ states and strongly hybridized, and also from localized completely filled $t_{2 g}$ states of the $\mathrm{Ni}$ ions.

In this paper, we show that despite these challenges, the cRPA method is indeed able to produce the large renormalization of the Coulomb repulsion $U$. We also show that $U_{\sigma \sigma}$ is further effectively reduced due to intersite Coulomb interactions down to values comparable to the Peierls gap $\Delta_{S}$, hence establishing on firm grounds the low-energy description suggested previously $[13,14]$, with the additional twist of large nonlocal interactions effectively renormalizing the local ones. We calculate the phase diagram of $\mathrm{LuNiO}_{3}$ within a combination of density-functional-theory-based electronic structure and dynamical mean-field theory (DFT+DMFT), including the intersite interactions at a static mean-field level. For the cRPA values of $U, J$ and of the intersite interactions, our DFT+DMFT calculations yield a metallic state for the orthorhombic phase and a disproportionated insulator for the monoclinic one, in agreement with experiments.

This paper is organized as follows. In Sec. II, we provide an introduction to the electronic structure of $\mathrm{LuNiO}_{3}$ and to the effective low-energy description in terms of $e_{g}$ states. In Sec. III, we implement the constrained random-phase approximation and compute the resulting $a b$ initio interaction parameters. In Sec. IV, we summarize the $a b$ initio construction of the low-energy effective model and explore its phase diagram within the DFT+DMFT framework for both the orthorhombic and monoclinic phases, as a function of $U$ and $J$. We show that the cRPA-calculated values of these parameters correspond to a location of each of the two structures in this phase diagram, which is physically consistent. Our results and findings are briefly summarized and discussed in Sec. V.

\section{ELECTRONIC STRUCTURE AND LOW-ENERGY MODEL}

The electronic structure of both the low-temperature monoclinic (space group $P 2_{1} / n$; see Fig. 2) and high-temperature orthorhombic ( $P$ bnm $)$ phases of $\mathrm{LuNiO}_{3}$ have been calculated using the experimental lattice structures provided in Ref. [35] $(a, b, c=5.132,5.529,7.344 \AA$ at $T=673 \mathrm{~K}$ for $P b n m$ and $a, b, c=5.124,5.509,7.355 \AA$ at $T=533 \mathrm{~K}$ for $\left.P 2_{1} / n\right)$. The unit cells of both structures contain four formula units, but the monoclinic one differs by having two distinct types of $\mathrm{NiO}_{6}$ octahedra: one with short $\mathrm{Ni}-\mathrm{O}$ bonds and one with long bonds corresponding to compressed and expanded octahedra. For the reader's convenience, we list the fractional coordinates of the $\mathrm{Ni}$ sites in the monoclinic cell in Table I. 


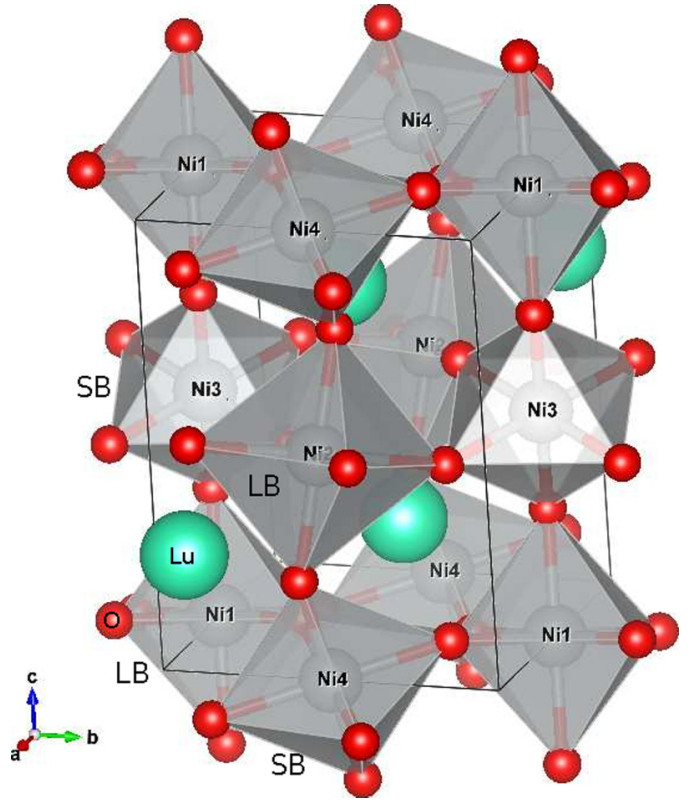

FIG. 2. $\mathrm{LuNiO}_{3}$ in the monoclinic phase $\left(P 2_{1} / n\right.$ with the $a^{-} a^{-} c^{+}$ octahedra rotation pattern in Glazer notation). Oxygen atoms (red) form distorted octahedra containing $\mathrm{Ni}$ (gray). This structure is intercalated with $\mathrm{Lu}$ atoms (turquoise). The short-bond (SB) and long-bond (LB) octahedra are identified. The labels of the Ni atoms correspond to the positions given in Table I.

In our density-functional-theory (DFT) calculations within the local-density approximation (LDA), we have employed the full-potential augmented-plane-wave (FLAPW) method as implemented in the FLEUR package [36,37]. All calculations were performed using a $\mathbf{k}$ mesh consisting of $4 \times 4 \times 2$ points.

The calculated low-energy band structure of monoclinic $\mathrm{LuNiO}_{3}$ (top panel of Fig. 3) features a manifold of eight $e_{g}$ bands in the range of $[-0.4: 1.9] \mathrm{eV}$ around the Fermi level, with the filled $t_{2 g}$ bands located below $-0.7 \mathrm{eV}$ in energy and, hence, well separated from the $e_{g}$ ones. We note that a small "Peierls gap," $\Delta_{s}^{\mathrm{DFT}} \simeq 0.25 \mathrm{eV}$, separates the $e_{g}$ bands into two sets of four bands (except an isolated point $U$ ), at an energy corresponding to the nominal filling of two electrons per site (half filling), i.e., about $+0.5 \mathrm{eV}$ above the LDA Fermi level. The Peierls gap originates from the existence of two types of sites in the distorted monoclinic phase: LB sites are pushed down in energy relative to the more covalent SB sites, for

TABLE I. Fractional coordinates and types of $\mathrm{Ni}$ sites in the monoclinic structure of $\mathrm{LuNiO}_{3} . \mathrm{Ni}_{1}$ and $\mathrm{Ni}_{2}$ are of the $\mathrm{LB}$ type and lie diagonally across from each other, as do the $\mathrm{SB}$ sites $\mathrm{Ni}_{3}$ and $\mathrm{Ni}_{4}$. $\mathrm{Ni}_{1}$ has as nearest neighbors $2 \times \mathrm{Ni}_{3}$ in the $z$ direction and $4 \times \mathrm{Ni}_{4}$ in the $x-y$ plane.

\begin{tabular}{lllll}
\hline \hline & Type & $X$ & $Y$ & $Z$ \\
\hline $\mathrm{Ni}_{1}$ & LB & $\frac{1}{2}$ & 0 & 0 \\
$\mathrm{Ni}_{2}$ & LB & 0 & $\frac{1}{2}$ & $\frac{1}{2}$ \\
$\mathrm{Ni}_{3}$ & SB & $\frac{1}{2}$ & 0 & $\frac{1}{2}$ \\
$\mathrm{Ni}_{4}$ & SB & 0 & $\frac{1}{2}$ & 0 \\
\hline \hline
\end{tabular}
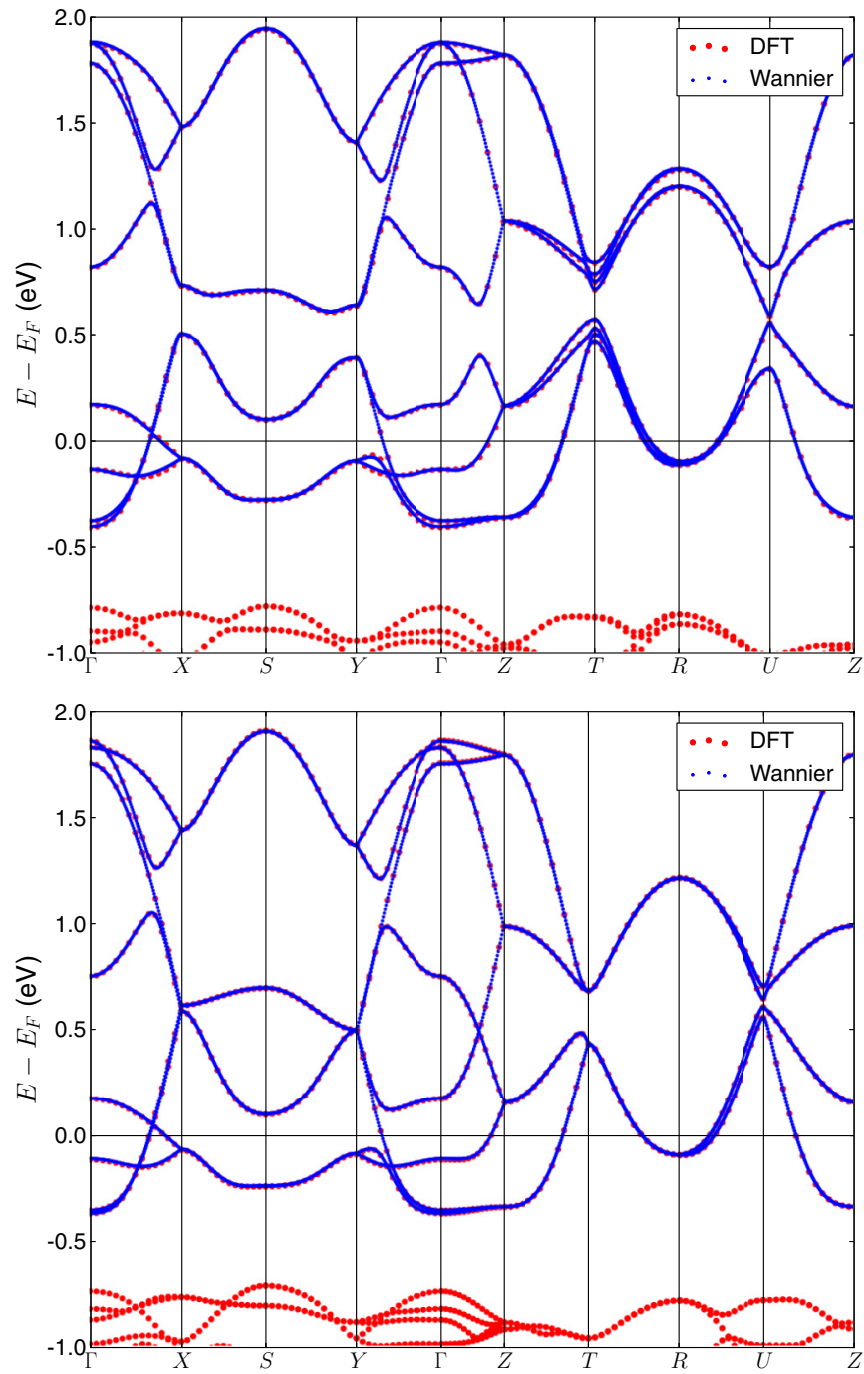

FIG. 3. The band structure of the monoclinic (top) and orthorhombic (bottom) phases of $\mathrm{LuNiO}_{3}$ for a unit cell consisting of four formula units. The DFT bands are shown in red and the band structure produced by the Wannier-basis low-energy Hamiltonian is shown in blue. Within the numerical accuracy, the Wannier bands are identical to the DFT $e_{g}$ bands. The Peierls gap in the monoclinic phase splits the set of eight $e_{g}$ bands into two separated sets of four bands.

which the Ni $d$ and $\mathrm{O} p$ orbitals overlap more. The DFT band structure of the orthorhombic phase is quite similar to that of the monoclinic phase (bottom panel of Fig. 3) apart from the fact that the Peierls gap is, of course, closed in this case.

In order to construct the quadratic part of the low-energy model, we downfold the states around the Fermi energy. A set of maximally localized Wannier functions representing correlated $e_{g}$ states on $\mathrm{Ni}$ sites was constructed from the eight $e_{g}$ bands using the WANNIER90 package [38]. Given the absence of entanglement between the $e_{g}$ bands and the rest of the electronic structure in $\mathrm{LuNiO}_{3}$, the projection from the space of Kohn-Sham $e_{g}$ eigenstates onto the Wannier basis is simply a unitary transformation. Hence, by diagonalizing the resulting low-energy Wannier Hamiltonian, one reproduces the original DFT bands, as shown in Fig. 3. 


\section{CONSTRAINED RANDOM-PHASE APPROXIMATION}

\section{A. Method description}

With the quadratic part of the low-energy Hamiltonian for the $e_{g}$ states, we perform ab initio calculations of the corresponding interaction terms using the constrained randomphase approximation (cRPA) [27].

The main idea behind the cRPA is to write the effective interactions suitable for a low-energy multiband model as the matrix elements of a partially screened interaction in the chosen localized basis. Calculations within the cRPA method start from the evaluation of the bare (unscreened) Coulomb vertex $v$. The partial polarization function $P_{r}(\omega)$ is then calculated. This describes the screening of $v$ within RPA by including all particle-hole transitions, except those within the low-energy $e_{g}$ subspace, i.e., $P_{r}(\omega)=P(\omega)-P_{e_{g}}(\omega)$, where $P(\omega)$ is the total polarization function and $P_{e_{g}}(\omega)$ is the contribution of all transitions within the low-energy subspace. Processes within the low-energy subspace will be subsequently treated explicitly by solving the low-energy Hamiltonian with more sophisticated many-body techniques beyond the RPA, such as DMFT.

The partially screened frequency-dependent interaction is obtained as $W_{r}(\omega)=v /\left[1-P_{r}(\omega) v\right]$. The Hubbard interactions are then obtained from matrix elements of the static limit $W_{r}(\omega=0)$ of this effective interaction. An important advantage of the cRPA is its ability to easily treat intersite interactions, as demonstrated in Refs. [39-41].

The cRPA procedure is unambiguously defined when a subset of correlated states is separated from the rest of the bands, as is the case with the $e_{g}$ bands of $\mathrm{LuNiO}_{3}$. However, in the case of an entanglement between correlated and uncorrelated bands, one faces the problem of determining which screened processes should be included in $P_{r}(\omega)$. The two schemes that have been proposed to date to handle this issue, i.e., disentanglement [42] and projection [43], both give identical results in our case with $P_{e_{g}}(\omega)$ including all transitions within the $e_{g}$ manifold, as the eight $e_{g}$ bands are well separated from all other bands.

In our calculations, the on-site and intersite effective interactions between Ni $e_{g}$ states are obtained using the cRPA functionality of the SPEX code, a GW code based on the FLEUR [36] electronic structure package [44]. There were 800 Kohn-Sham bands included, up to the energy cutoff of $140 \mathrm{eV}$ in $P(\omega)$ and, correspondingly, $P_{r}(\omega)$. In order to correctly describe semicore and high-energy Kohn-Sham states, we extended the FLAPW basis by including additional local orbitals $[45,46]$. Namely, we included local orbitals for the $3 s, 4 s, 5 s, 3 p, 4 p, 5 p, 3 d, 4 d, 4 f$, and $5 f$ shells of Ni; $2 s, 3 s$, $2 p, 3 p, 3 d, 4 d, 4 f$, and $5 f$ shells of $\mathrm{O} ; 5 s, 6 s, 7 s, 5 p, 6 p, 7 p$, $5 d, 6 d, 4 f$, and $5 f$ shells of $\mathrm{Lu}$; as well as $6 g$ and $7 h$ shells on all atoms.

The cRPA scheme gives access to the fully frequencydependent interactions and, in principle, it is possible to perform many-body calculations including this dynamical character of the interactions [47-49]. In the low-energy electronic structure, the main consequence of dynamical screening is a renormalization of the single-particle hopping parameters by a renormalization factor that can by explicitly calculated once $\mathcal{U}(\omega)$ is known [47]. It has been argued, however, that in real materials, partial cancellations of this renormalization with band widening effects by nonlocal exchange occur [50,51], and including one but not the other leads to inconsistencies. A consistent treatment of both together can naturally be obtained within combined many-body perturbation theory and dynamical mean-field theory schemes, e.g., in the so-called GW+DMFT scheme [52] or simplified variants [49,53], and recent materials studies have both highlighted the cancellation effects [51,54,55] and subtle Fermi-surface renormalizations going beyond [49]. Since in the present work, however, we are mainly interested in the energetics of charge ordering phenomena that should not depend on the fine details of the band dispersions, we disregard both effects, relying on the overall cancellation effects between dynamical screening and nonlocal (exchange) self-energy contributions evidenced in the literature. Finally, one may wonder about the validity of the cRPA for the calculation of the static Hubbard interactions, given that it reduces polarization effects to particle-hole bubble diagrams neglecting any vertex effects. Several recent works have addressed this question based on different strategies: Ref. [56] proposed a self-screening corrected version of the cRPA, analogously to the self-screening corrected GW scheme of Ref. [57]. These authors moreover investigate the accuracy of the cRPA for a three-band model with on-site interactions. Since they do not include hybridizations, however, the bands effectively decouple and the bare interaction turns out to be the optimal value for the effective interaction. These authors further note the difficulty to extend the study to more realistic models since those should include both a substantially larger number of screening degrees of freedom to simulate the extended environment in a solid and the long-range interactions present in realistic materials. An alternative strategy of assessing the limitations of the cRPA has been presented by Kinza and Honerkamp [58]. In this work, functional renormalization-group techniques are used to include, in a systematic diagrammatic way, corrections to the cRPA contributions. While the difficulty to go to sufficiently realistic models to compare with real materials problems also persists here, an interesting result of this work stems from an analysis of the role of symmetries: the authors argue that in systems with different symmetries of target bands and screening bands, corrections beyond cRPA are small since certain classes of diagrams are suppressed by selection rules. In the present case, this situation is indeed realized since our target bands are dominantly of $e_{g}$ character, while screening bands consist of non- $e_{g}$ states. While giving quantitative error bars for cRPA calculations thus remains difficult, these arguments nevertheless suggest that cRPA is fully justified for our purposes.

\section{B. cRPA results for $\mathrm{LuNiO}_{3}$}

Here we discuss the interaction parameters for the lowenergy models as obtained by cRPA. We consider only the density-density interaction terms for parallel and antiparallel spins. Note that the coupling constants $J_{X}$ and $J_{P}$, corresponding respectively to the spin-flip and pair-hopping terms, can be obtained from the density-density terms under the assumption of rotational invariance. All results are given in the basis of the eight $e_{g}$ orbitals, ordered as $z^{2}$ and $x^{2}-y^{2}$ for each of the 
four $\mathrm{Ni}$ sites as given above. The results for the orthorhombic phase are given in Table II and those for the monoclinic phase are given in Table III.

\section{Orthorhombic $\mathrm{LuNiO}_{3}$}

For the orthorhombic phase of $\mathrm{LuNiO}_{3}$, the average densitydensity interaction between electrons with opposite spins in the same orbital is found to be $U=1.65 \mathrm{eV}$, and that between opposite spins in different orbitals is $U^{\prime}=0.99 \mathrm{eV}$. The interaction between parallel spins in different orbitals is the smallest, in accordance with Hund's rule, being reduced to $U^{\sigma \sigma}=U^{\prime}-J=0.66 \mathrm{eV}$. We observe that despite the orthorhombic distortion, these parameters almost perfectly obey the relation $U^{\prime}=U-2 J$ expected for a cubic system, with $U=1.65 \mathrm{eV}, J=0.33 \mathrm{eV}$.

The average nearest-neighbor parallel-spin interaction $V_{1}^{\sigma \sigma}$ is $0.42 \mathrm{eV}$, where the average is taken evenly over both neighbors in the unit cell, comparable to the average on-site parallel-spin interaction $U^{\prime}-J=0.66 \mathrm{eV}$. Additionally, the next-nearest-neighbor parallel-spin interaction $V_{2}^{\sigma \sigma}$ can be estimated from, e.g., $\mathrm{Ni}_{1}$ and $\mathrm{Ni}_{2}$, to be $0.30 \mathrm{eV}$.

\section{Monoclinic $\mathrm{LuNiO}_{3}$}

For the monoclinic phase, we obtain for the averaged parameters $U=1.83, U^{\prime}=1.09$, and $U^{\prime}-J=0.74 \mathrm{eV}$, which is fairly consistent with the Kanamori parametrization for a cubic system with $U=1.83, J=0.37 \mathrm{eV}$ 's.

The on-site parallel-spin interaction $U^{\sigma \sigma}=U-3 J=$ $0.74 \mathrm{eV}$ is again of a similar order of magnitude to the average nearest-neighbor parallel-spin interaction $V_{1}^{\sigma \sigma}=0.44 \mathrm{eV}$. In this case, due to the distortions in the structure, the average needs to be weighted to account for the fact that $\mathrm{Ni}_{1}$ has four $\mathrm{Ni}_{4}$ atoms and two $\mathrm{Ni}_{3}$ atoms as neighbors. For the next-nearest-neighbor interaction, we obtain $V_{2}^{\sigma \sigma}=0.31 \mathrm{eV}$.

\section{Long-range nature of interactions}

For both phases, we notice that the nearest-neighbor intersite interactions $V_{1}$, for example between $\mathrm{Ni}_{1}$ and $\mathrm{Ni}_{3}$ and between $\mathrm{Ni}_{1}$ and $\mathrm{Ni}_{4}$, are found to be non-negligible. It must be emphasized that the contributions to screening from particlehole transitions within the $e_{g}$ manifold of bands are excluded in the cRPA procedure. Indeed, the effective interactions obtained from cRPA are to be used in the low-energy effective $e_{g}$ model, and further screening relies on the many-body treatment of this model (e.g., with DMFT). As a result, the interactions in the low-energy effective model are screened exclusively by interband transitions, as in an insulator, and thus one should expect significant long-range Coulomb interactions. Indeed, the second-nearest-neighbor interactions $V_{2}$ are likewise quite large. Upon closer inspection, it is clear that the interactions up to the second shell of neighbors decay as $1 / R$, indicating that long-range interactions must be accounted for to reach an accurate description of the physics of these materials. The situation is comparable to the recently studied case of $\mathrm{Sn} / \mathrm{Si}(111)$ [39-41]: there it was shown that the continuum limit that allows one to parametrize the interaction tail as $\frac{V_{1}}{R}$ with the nearest-neighbor interaction $V_{1}=\epsilon^{-1} V_{1}^{\text {bare }}$, with $\epsilon$ the macroscopic dielectric constant, is already reached at nearest-neighbor distances. Similarly, in graphene, the long- range tail of the interactions was argued to be responsible for the necessary screening to prevent the system from becoming a Mott insulator [59]. More generally, the effects of nonlocal interactions in correlated materials and models thereof have recently raised tremendous interest in the community [23,24,26,60-67], within different lattice geometries.

From Tables II and III, one may also conclude that the spin dependence of intersite interactions is negligible; the exchange interaction arises due to direct overlap of the $e_{g}$ orbitals and, therefore, is well localized. Hence, from now on, we will suppress the spin subscripts in the intersite interactions $V$.

\section{PHYSICAL CONSEQUENCES FOR $\mathrm{LuNiO}_{3}$ AND DMFT CALCULATIONS}

\section{A. Effective theory for low-energy $e_{g}$ states}

From the sections above, we can infer the following effective Hamiltonian for a description of $\mathrm{LuNiO}_{3}$ involving only low-energy $e_{g}$ states:

$$
\hat{H}=\hat{H}_{0}+\sum_{i} \hat{H}_{U}^{(i)}+\hat{H}_{V}
$$

In this expression, $\hat{H}_{0}$ is the single-electron part of the effective Hamiltonian. Within the DFT+DMFT framework, $\hat{H}_{0}$ is constructed as

$$
\hat{H}_{0}=\hat{H}_{\mathrm{DFT}}^{0}-\hat{H}_{\mathrm{dc}},
$$

with $\hat{H}_{\mathrm{DFT}}^{0}$ the single-electron Kohn-Sham Hamiltonian for $e_{g}$ bands, as obtained from DFT(-LDA) and $\hat{H}_{\mathrm{dc}}$ is a doublecounting correction to be detailed below. The many-body terms $\hat{H}_{U}$ and $\hat{H}_{V}$ are local (on-site) and intersite interaction terms, respectively. For the local term, the full Kanamori Hamiltonian appropriate for $e_{g}$ states is considered, namely, on each lattice site $(i)$ :

$$
\begin{aligned}
\hat{H}_{U}= & U \sum_{m} \hat{n}_{m \uparrow} \hat{n}_{m \downarrow}+(U-2 J) \sum_{m \neq m^{\prime}} \hat{n}_{m \uparrow} \hat{n}_{m^{\prime} \downarrow} \\
& +(U-3 J) \sum_{m<m^{\prime}, \sigma} \hat{n}_{m \sigma} \hat{n}_{m^{\prime} \sigma} \\
& -J \sum_{m \neq m^{\prime}} c_{m \uparrow}^{\dagger} c_{m \downarrow} c_{m^{\prime} \downarrow}^{\dagger} c_{m^{\prime} \uparrow}+J \sum_{m \neq m^{\prime}} c_{m \uparrow}^{\dagger} c_{m \downarrow}^{\dagger} c_{m^{\prime} \downarrow} c_{m^{\prime} \uparrow} \cdot
\end{aligned}
$$

The intersite term is taken to be of the form

$$
\hat{H}_{V}=\frac{1}{2} \sum_{i \neq j} V_{i j} \hat{n}_{i} \hat{n}_{j} .
$$

In this expression, the coupling constants $U, J$, and $V_{i j}=$ $V_{1} / R_{i j}$ (with $R_{i j}$ the distance between the two atomic sites $i, j$ ) are determined from the cRPA calculations presented above.

In the following, we show that this low-energy effective model with cRPA values of the interaction parameters provides a satisfactory description of the physics of $\mathrm{LuNiO}_{3}$. This is done by using the DFT+DMFT framework in order to construct and solve the low-energy model. We find that intersite interactions must be taken into account in this lowenergy description: they are included in our calculations at the level of Hartree mean-field theory. Finally, these findings are discussed in relation to the low-energy picture of rare-earth nickelates proposed in Ref. [13]. 
TABLE II. $U_{m m^{\prime}}$ for antiparallel and parallel spins for orthorhombic $\mathrm{LuNiO}_{3}$. All values in eV. Two-index parameters are related to four-index ones as $U_{m m^{\prime}}^{\sigma \bar{\sigma}}=U_{m m^{\prime} m m^{\prime}}, U_{m m^{\prime}}^{\sigma \sigma}=U_{m m^{\prime} m m^{\prime}}-U_{m m^{\prime} m^{\prime} m}$.

\begin{tabular}{|c|c|c|c|c|c|c|c|}
\hline \multicolumn{8}{|c|}{$U_{m m^{\prime}}^{\sigma \bar{\sigma}}($ antiparallel spin $)$} \\
\hline $\mathrm{Ni}_{1}, z^{2}$ & $\mathrm{Ni}_{1}, x^{2}-y^{2}$ & $\mathrm{Ni}_{2}, z^{2}$ & $\mathrm{Ni}_{2}, x^{2}-y^{2}$ & $\mathrm{Ni}_{3}, z^{2}$ & $\mathrm{Ni}_{3}, x^{2}-y^{2}$ & $\mathrm{Ni}_{4}, z^{2}$ & $\mathrm{Ni}_{4}, x^{2}-y^{2}$ \\
\hline 1.77 & 0.99 & 0.32 & 0.30 & 0.62 & 0.43 & 0.39 & 0.42 \\
\hline 0.99 & 1.54 & 0.30 & 0.27 & 0.43 & 0.33 & 0.40 & 0.45 \\
\hline 0.32 & 0.30 & 1.77 & 0.99 & 0.39 & 0.40 & 0.62 & 0.43 \\
\hline 0.30 & 0.27 & 0.99 & 1.54 & 0.42 & 0.45 & 0.43 & 0.33 \\
\hline 0.62 & 0.43 & 0.39 & 0.42 & 1.77 & 0.99 & 0.32 & 0.30 \\
\hline 0.43 & 0.33 & 0.40 & 0.45 & 0.99 & 1.54 & 0.30 & 0.27 \\
\hline 0.39 & 0.40 & 0.62 & 0.43 & 0.32 & 0.30 & 1.77 & 0.99 \\
\hline 0.42 & 0.45 & 0.43 & 0.33 & 0.30 & 0.27 & 0.99 & 1.54 \\
\hline \multicolumn{8}{|c|}{$U_{m m^{\prime}}^{\sigma \sigma}($ parallel spin $)$} \\
\hline $\mathrm{Ni}_{1}, z^{2}$ & $\mathrm{Ni}_{1}, x^{2}-y^{2}$ & $\mathrm{Ni}_{2}, z^{2}$ & $\mathrm{Ni}_{2}, x^{2}-y^{2}$ & $\mathrm{Ni}_{3}, z^{2}$ & $\mathrm{Ni}_{3}, x^{2}-y^{2}$ & $\mathrm{Ni}_{4}, z^{2}$ & $\mathrm{Ni}_{4}, x^{2}-y^{2}$ \\
\hline 0.00 & 0.66 & 0.32 & 0.30 & 0.59 & 0.42 & 0.38 & 0.41 \\
\hline 0.66 & 0.00 & 0.30 & 0.27 & 0.42 & 0.33 & 0.39 & 0.42 \\
\hline 0.32 & 0.30 & 0.00 & 0.66 & 0.38 & 0.39 & 0.59 & 0.42 \\
\hline 0.30 & 0.27 & 0.66 & 0.00 & 0.41 & 0.42 & 0.42 & 0.33 \\
\hline 0.59 & 0.42 & 0.38 & 0.41 & 0.00 & 0.66 & 0.32 & 0.30 \\
\hline 0.42 & 0.33 & 0.39 & 0.42 & 0.66 & 0.00 & 0.30 & 0.27 \\
\hline 0.38 & 0.39 & 0.59 & 0.42 & 0.32 & 0.30 & 0.00 & 0.66 \\
\hline 0.41 & 0.42 & 0.42 & 0.33 & 0.30 & 0.27 & 0.66 & 0.00 \\
\hline
\end{tabular}

\section{B. Hartree treatment of long-range interactions}

In order to take the intersite terms into account, we employ the Hartree approximation for long-range interactions, which provides a leading contribution to the self-energy in the disproportionated phase. Note that this is consistent with the DMFT approach, in which only local interactions have dynamical effects, while nonlocal interactions are treated at the static mean-field level. One might argue that dynamical effects beyond DMFT, as included in, e.g., the extended dynamical mean-field theory (EDMFT), could also be important for this system. Indeed, it is well known that dynamical fluctuations can give substantial deviations from the static mean-field treatment of intersite interactions. However, these mainly appear in the regime of stronger coupling where the charge order transition emerges from the Mott insulating phase, while - at least at the level of a simple extended Hubbard model-deviations are small in the regime of weaker coupling (see Fig. 8 of Ref. [67]).

TABLE III. $U_{m m^{\prime}}$ for antiparallel and parallel spins for monoclinic $\mathrm{LuNiO}_{3}$. All values in eV. Two-index parameters are related to four-index ones as $U_{m m^{\prime}}^{\sigma \bar{\sigma}}=U_{m m^{\prime} m m^{\prime}}, U_{m m^{\prime}}^{\sigma \sigma}=U_{m m^{\prime} m m^{\prime}}-U_{m m^{\prime} m^{\prime} m}$.

\begin{tabular}{|c|c|c|c|c|c|c|c|}
\hline \multicolumn{8}{|c|}{$U_{m m^{\prime}}^{\sigma \bar{\sigma}}($ antiparallel spin $)$} \\
\hline $\mathrm{Ni}_{1}, z^{2}$ & $\mathrm{Ni}_{1}, x^{2}-y^{2}$ & $\mathrm{Ni}_{2}, z^{2}$ & $\mathrm{Ni}_{2}, x^{2}-y^{2}$ & $\mathrm{Ni}_{3}, z^{2}$ & $\mathrm{Ni}_{3}, x^{2}-y^{2}$ & $\mathrm{Ni}_{4}, z^{2}$ & $\mathrm{Ni}_{4}, x^{2}-y^{2}$ \\
\hline 1.73 & 1.07 & 0.32 & 0.31 & 0.63 & 0.46 & 0.39 & 0.44 \\
\hline 1.07 & 1.88 & 0.31 & 0.29 & 0.45 & 0.37 & 0.43 & 0.50 \\
\hline 0.32 & 0.31 & 1.73 & 1.07 & 0.39 & 0.42 & 0.63 & 0.46 \\
\hline 0.31 & 0.29 & 1.07 & 1.88 & 0.44 & 0.50 & 0.45 & 0.37 \\
\hline 0.63 & 0.45 & 0.39 & 0.44 & 1.82 & 1.12 & 0.32 & 0.31 \\
\hline 0.46 & 0.37 & 0.42 & 0.50 & 1.12 & 1.89 & 0.31 & 0.29 \\
\hline 0.39 & 0.43 & 0.63 & 0.45 & 0.32 & 0.31 & 1.82 & 1.12 \\
\hline 0.44 & 0.50 & 0.46 & 0.37 & 0.31 & 0.29 & 1.12 & 1.89 \\
\hline \multicolumn{8}{|c|}{$U_{m m^{\prime}}^{\sigma \sigma}($ parallel spin $)$} \\
\hline $\mathrm{Ni}_{1}, z^{2}$ & $\mathrm{Ni}_{1}, x^{2}-y^{2}$ & $\mathrm{Ni}_{2}, z^{2}$ & $\mathrm{Ni}_{2}, x^{2}-y^{2}$ & $\mathrm{Ni}_{3}, z^{2}$ & $\mathrm{Ni}_{3}, x^{2}-y^{2}$ & $\mathrm{Ni}_{4}, z^{2}$ & $\mathrm{Ni}_{4}, x^{2}-y^{2}$ \\
\hline 0.00 & 0.74 & 0.32 & 0.31 & 0.60 & 0.45 & 0.38 & 0.43 \\
\hline 0.74 & 0.00 & 0.31 & 0.29 & 0.45 & 0.37 & 0.42 & 0.48 \\
\hline 0.32 & 0.31 & 0.00 & 0.74 & 0.38 & 0.42 & 0.60 & 0.45 \\
\hline 0.31 & 0.29 & 0.74 & 0.00 & 0.43 & 0.48 & 0.45 & 0.37 \\
\hline 0.60 & 0.45 & 0.38 & 0.43 & 0.00 & 0.83 & 0.32 & 0.31 \\
\hline 0.45 & 0.37 & 0.42 & 0.48 & 0.83 & 0.00 & 0.31 & 0.29 \\
\hline 0.38 & 0.42 & 0.60 & 0.45 & 0.32 & 0.31 & 0.00 & 0.82 \\
\hline 0.43 & 0.48 & 0.45 & 0.37 & 0.31 & 0.29 & 0.82 & 0.00 \\
\hline
\end{tabular}


In the Hartree approximation, $\hat{H}_{V}$ reduces to

$$
\hat{H}_{V} \rightarrow \frac{1}{2} \sum_{i \neq j} V_{i j}\left[n_{i} \hat{n}_{j}+n_{j} \hat{n}_{i}-n_{i} n_{j}\right],
$$

with the effective Hartree one-body Hamiltonian and potential

$$
\hat{H}_{\text {eff }}=\sum_{i} V_{H}(i) \hat{n}_{i}, \quad V_{H}(i)=\sum_{j \neq i} V_{i j} n_{j} .
$$

The total energy from the interacting part of the Hamiltonian is thus

$$
E_{V}\left[\left\{n_{i}\right\}\right]=\frac{1}{2} \sum_{i \neq j} V_{i j} n_{i} n_{j}=\frac{1}{2} \sum_{i} V_{H}(i) n_{i} .
$$

Let us now consider the present case of the Ni sublattice in $\mathrm{LuNiO}_{3}$. It can be well approximated by the NaCl-type bipartite lattice with two inequivalent, LB and SB, sublattices with occupancies $n_{\mathrm{LB}}$ and $n_{\mathrm{SB}}$ per site, respectively. Given that the system is charge neutral, the formally diverging term on the right-hand side of Eq. (3) can be summed using the Madelung method, resulting in the following sublattice potentials:

$$
\begin{aligned}
& V_{H}(\mathrm{LB})=-M V_{1}\left(n_{\mathrm{LB}}-n_{\mathrm{SB}}\right) / 2, \\
& V_{H}(\mathrm{SB})=+M V_{1}\left(n_{\mathrm{LB}}-n_{\mathrm{SB}}\right) / 2,
\end{aligned}
$$

where $M$ is the Madelung constant for the $\mathrm{NaCl}$ lattice and the uniform part of the potential is dropped.

By comparing this result with the Hartree term with only nearest-neighbor interactions,

$$
\begin{aligned}
& V_{H}^{n n}(\mathrm{LB})=-z_{\mathrm{eff}} V_{1}\left(n_{\mathrm{LB}}-n_{\mathrm{SB}}\right) / 2, \\
& V_{H}^{n n}(\mathrm{SB})=+z_{\mathrm{eff}} V_{1}\left(n_{\mathrm{LB}}-n_{\mathrm{SB}}\right) / 2,
\end{aligned}
$$

we can identify $M$ as an effective connectivity of the lattice

$$
z_{\text {eff }}=M \approx 1.747 \text {. }
$$

Note that the effect of the Madelung summation is that the effective connectivity $z_{\text {eff }}$ is significantly reduced as compared to the lattice connectivity $z=6$.

The Hartree potential above can be viewed as a sitedependent contribution to the self-energy coming from the intersite interactions, which reads

$$
\Sigma_{\alpha}^{V}=z_{\mathrm{eff}} V_{1}\left(n_{\bar{\alpha}}-n_{\alpha}\right) / 2 \text {. }
$$

In this expression, $\bar{\alpha}$ designates the opposite sublattice relative to $\alpha$, i.e., if $\alpha=\mathrm{SB}$, then $\bar{\alpha}=\mathrm{LB}$, and vice versa; $n_{\alpha}$ is the $e_{g}$ occupancy of the corresponding site.

\section{Atomic limit}

Before discussing the DFT+DMFT results, we first consider the atomic limit in which all hopping terms in the Hamiltonian (1) are set to zero so that $\hat{H}_{0}$ contains only an onsite Peierls potential equal to $-\Delta_{s} / 2$ on LB sites and $+\Delta_{s} / 2$ on SB sites (see Fig. 1). We will compare in this limit the energies of two states: the uniform one (UN) $n_{\mathrm{LB}}=n_{\mathrm{SB}}=1$ and the fully disproportionated one (FD) $n_{\mathrm{LB}}=2, n_{\mathrm{SB}}=0$.

The contribution of the on-site Peierls potential to the energy is $-\Delta_{s} \sum_{i \in \mathrm{LB}} n_{i} / 2+\Delta_{s} \sum_{i \in \mathrm{SB}} n_{i} / 2$ : it vanishes in the uniform state and provides an energy gain $-\Delta_{s}\left(N_{s} / 2\right)$ in the FD state, with $N_{s}$ the total number of lattice sites. The on-site interaction energy vanishes too in the UN state, and is equal to $+U^{\sigma \sigma} N_{s} / 2$ in the FD state with $U^{\sigma \sigma}=U-3 J$, since two electrons on a LB site will occupy the high-spin Hund's rule configuration with one electron in each of the two $e_{g}$ orbitals. Finally, using the above expressions in the Hartree approximation, the contribution of the intersite interactions to the energy reads

$$
\left\langle\hat{H}_{V}\right\rangle=-\frac{N_{s}}{8} z_{\mathrm{eff}} V_{1}\left(n_{\mathrm{LB}}-n_{\mathrm{SB}}\right)^{2} .
$$

It vanishes again in the UN state and provides an energy gain $-V_{1} z_{\text {eff }} N_{s} / 2$ in the FD state. Hence, the energy difference between the FD state and the uniform one reads, in the atomic limit,

$$
\begin{aligned}
E_{\mathrm{FD}}-E_{\mathrm{UN}} & =\frac{N_{s}}{2}\left[U^{\sigma \sigma}-z_{\mathrm{eff}} V_{1}-\Delta_{s}\right] \\
& =\frac{N_{s}}{2}\left[U-3 J-z_{\mathrm{eff}} V_{1}-\Delta_{s}\right] .
\end{aligned}
$$

The transition into the charge-disproportionated state in the atomic limit occurs, therefore, when

$$
U^{\sigma \sigma}-z_{\text {eff }} V_{1}<\Delta_{s} .
$$

Note that if only the nearest-neighbor component of the nonlocal interactions is taken into account, the FD state is stable for $U^{\sigma \sigma}-z V_{1}<\Delta_{s}$. The above criterion in the presence of long-range interaction simply amounts to replacing the connectivity of the lattice $z$ by the effective Madelung connectivity.

Let us consider the orthorhombic phase where $\Delta_{s}=0$. The above criterion then reads $U-3 J-z_{\text {eff }} V_{1}<0$. Hence, a small enough value of $U$ (e.g., strongly reduced by screening) or a large enough value of the Hund's coupling $J$ leads to an instability into the disproportionated state, as noted in previous work $[13,14]$. In the present context, this instability is a spontaneous symmetry breaking of electronic origin, since all $\mathrm{Ni}$ sites are equivalent $\left(\Delta_{s}=0\right)$ in this crystal structure. Our cRPA results for $\mathrm{LuNiO}_{3}$ in the orthorhombic phase yield $U^{\sigma \sigma}-z_{\mathrm{eff}} V_{1} \simeq-0.1 \mathrm{eV}$ : the combined effect of screening and long-range interactions yields a small but negative value of this quantity, which is consistent with the physical picture of Subedi et al. [13]. Hence, surprisingly, in the atomic limit, we would conclude that the orthorhombic phase is spontaneously unstable to disproportionation. In reality, as shown below, the inclusion of intersite hopping in a full DFT+DMFT treatment leads to the correct conclusion that the orthorhombic phase is not electronically disproportionated-with the atomic-limit estimate providing a considerable overestimation of the range of stability of the FD state. However, inaccurate as it may be (especially in the metallic state), the virtue of this atomic-limit estimate is to emphasize how screening, a large $J$, and sizable intersite interactions can lead to disproportionation.

In the monoclinic phase, we obtained $U^{\sigma \sigma}=0.74 \mathrm{eV}$, $V_{1}=0.44 \mathrm{eV}$, and hence $U^{\sigma \sigma}-z_{\text {eff }} V_{1} \simeq-0.03 \mathrm{eV}$. Basically, any positive value of the Peierls energy gap, which is nonzero in this phase, will thus stabilize a fully disproportionated state in the atomic limit. As shown below, monoclinic $\mathrm{LuNiO}_{3}$ is indeed found to be a disproportionated insulator when performing DFT+DMFT calculations with these interaction parameters. 


\section{DMFT: Setup and double counting}

We now turn to the results obtained in the DFT+DMFT framework, first providing some technical details about the calculation.

The one-electron part of the effective Hamiltonian is $\hat{H}_{0}=\hat{H}_{\mathrm{DFT}}^{0}-\hat{H}_{\mathrm{dc}}$. The DFT Hamiltonian was obtained using the FLAPW method as implemented in the WIEN2K software package [68], with Perdew-Burke-Erzenhof (PBE) approximation [69] for the exchange-correlation functional. A $\mathbf{k}$ mesh of $6 \times 5 \times 4$ points is used. Projected local orbitals [70] spanning the low-energy $e_{g}$ subspace are constructed using the implementation of the TRIQS/DFTTOOLS software package [71-73].

The full local self-energy arises from both the DMFT treatment of the local interactions $H_{U}$ in (1) at the dynamical level and from the nonlocal interactions treated within the Hartree approximation, namely,

$$
\Sigma_{\alpha}\left(i \omega_{n}\right)=\Sigma_{\alpha}^{\mathrm{imp}}\left(i \omega_{n}\right)-\Sigma_{\mathrm{dc}, \alpha}^{\mathrm{imp}}+\Sigma_{\alpha}^{V}-\Sigma_{\mathrm{dc}, \alpha}^{V} .
$$

In this expression, $\alpha=\mathrm{LB}, \mathrm{SB}$ is an index labeling LB and $\mathrm{SB}$ sites, $\Sigma_{\alpha}^{V}$ is the Hartree self-energy,

$$
\Sigma_{\alpha}^{V}=z_{\mathrm{eff}} V_{1}\left(n_{\bar{\alpha}}-n_{\alpha}\right) / 2,
$$

and $\Sigma_{\alpha}^{\text {imp }}\left(i \omega_{n}\right)$ is obtained by solving the DMFT effective impurity model using the hybridization-expansion continuous-time quantum Monte Carlo (CTQMC) algorithm TRIQS/CTHYB [73,74].

A double-counting (DC) correction must be included in order to remove the contribution from interactions already included within DFT. This DC correction can be viewed equivalently as the $\hat{H}_{\text {dc }}$ part of $\hat{H}_{0}$ or as part of the self-energy. The DC correction to the self-energy arising from the $U, J$ interactions is evaluated in the fully localized limit [75] as follows [71]:

$$
\Sigma_{\mathrm{dc}, \alpha}^{\mathrm{imp}}=\bar{U}\left(n_{\alpha}^{\mathrm{DFT}}-1 / 2\right)-\bar{J}\left(n_{\alpha}^{\mathrm{DFT}} / 2-1 / 2\right),
$$

while the DC correction to the Hartree self-energy associated with the long-range interactions reads

$$
\Sigma_{\mathrm{dc}, \alpha}^{V}=z_{\mathrm{eff}} V_{1}\left(n_{\bar{\alpha}}^{\mathrm{DFT}}-n_{\alpha}^{\mathrm{DFT}}\right) / 2 .
$$

In these expressions, $\bar{U}=U-J$ is the average interaction between electrons with opposite spins, $\bar{J}=\bar{U}-U^{\sigma \sigma}=2 J$, and $n_{\alpha}^{\mathrm{DFT}}$ is the occupancy of the Ni $e_{g}$ shell in DFT for the site $\alpha=$ LB or SB.

In the monoclinic phase, the two inequivalent $\mathrm{Ni}$ sites have different $e_{g}$ occupancy already at the DFT level: $n_{\alpha}^{\mathrm{DFT}}=1.17$ and 0.83 for the LB and SB sites, respectively. From the expressions above, one sees that the Peierls energy splitting between LB and SB sites appearing in $\hat{H}_{0}=\hat{H}_{\mathrm{DFT}}^{0}-\hat{H}_{\mathrm{dc}}$ is renormalized by double counting, as compared to its DFT value,

$$
\Delta_{s}=\Delta_{s}^{\mathrm{DFT}}+\left(U-2 J-z_{\mathrm{eff}} V_{1}\right) \Delta n^{\mathrm{DFT}},
$$

with $\Delta n^{\mathrm{DFT}}=n_{\mathrm{LB}}^{\mathrm{DFT}}-n_{\mathrm{SB}}^{\mathrm{DFT}}$. Note that given the cRPA values above, $U-2 J-z_{\text {eff }} V_{1} \simeq 0.32 \mathrm{eV}$ is positive so that double counting enhances the effective value of the Peierls energy, from $\simeq 0.25 \mathrm{eV}$ at the DFT level to $\Delta_{s} \simeq 0.36 \mathrm{eV}$.
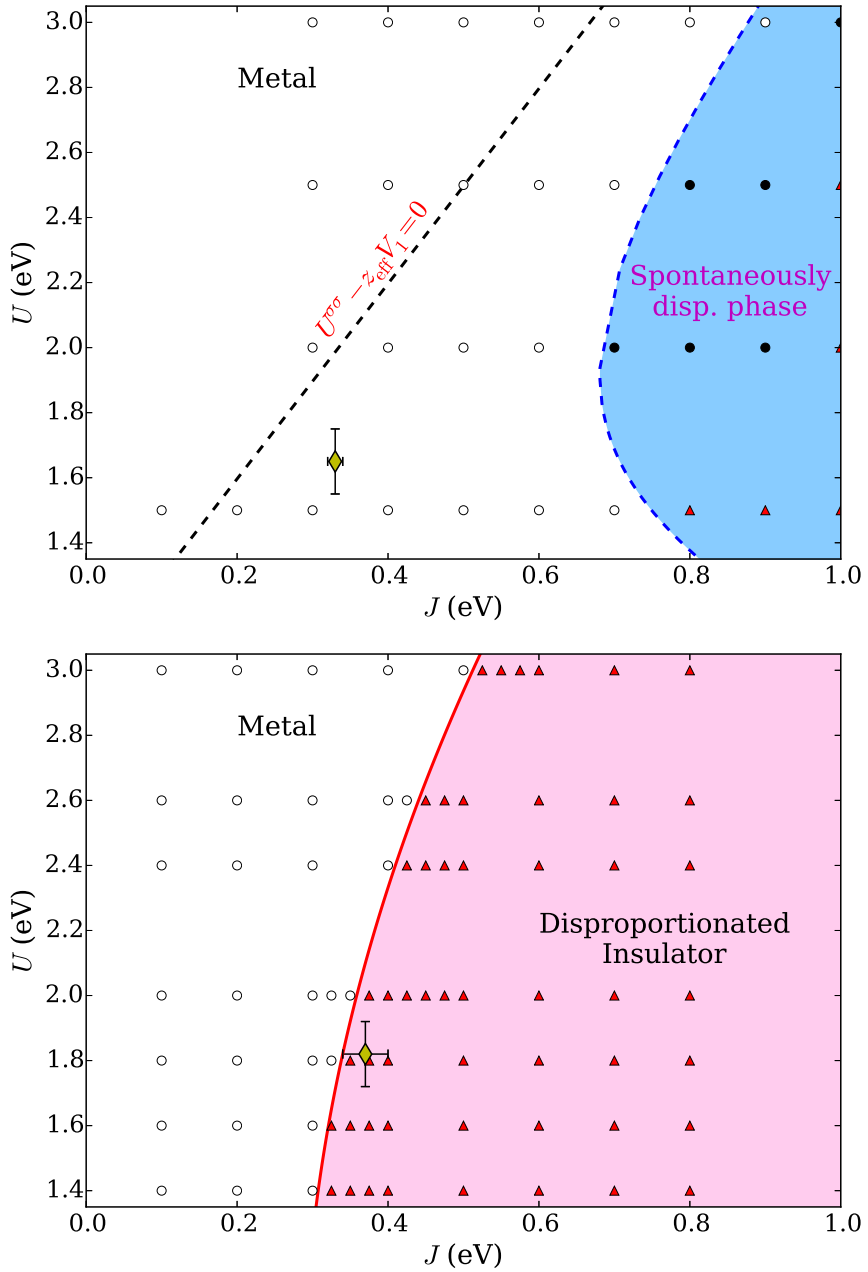

FIG. 4. Phase diagrams of the monoclinic (bottom) and orthorhombic (top) phases of $\mathrm{LuNiO}_{3}$. For the monoclinic phase: empty circles show metallic solutions, red triangles correspond to a disproportionated insulator. For the orthorhombic phase: empty circles stand for uniform metallic, filled circles show disproportionated metallic, and red triangles show disproportionated insulating solutions; the blue domain contains spontaneously disproportionated (metallic or insulating) solutions. The value of $V_{1}$ is fixed to the average cRPA value. The cRPA values of $U$ and $J$ are marked by the diamond, with error bars showing variations for different sites and orbitals.

\section{E. DMFT: Results and phase diagram}

In order to explore how the values of the interaction strengths affect the physics of $\mathrm{LuNiO}_{3}$ in each crystal structure, we have performed a series of DFT+DMFT calculations for a fixed value of $V_{1}=0.44 \mathrm{eV}$ with varying $U$ and $J$. The obtained phase diagrams are presented in Fig. 4. The main qualitative features are similar to the results of Ref. [13], in which the nonlocal interactions were not taken into account and only the local Kanamori interactions were included. Specifically, both the monoclinic and orthorhombic structures have a phase boundary separating a uniform metallic and a disproportionated (insulating or metallic) phase. The major quantitative difference from the earlier results is a considerable shift of the phase boundaries to the left in the present case, which is a direct consequence of the intersite coupling. This is 
consistent with the renormalization by $V_{1}$ of the atomic-limit stability criterium, given by Eq. (7) (dashed line in the top panel of Fig. 4), which can be compared to Eq. (4) in Ref. [13].

We note that the location of this boundary is very different for the orthorhombic and for the monoclinic phase, being pushed towards much smaller values of $J$ for the latter. This demonstrates the strong sensitivity of the disproportionation to the value of the Peierls energy [13].

The physical range of interaction parameters must be associated with regions of the phase diagram corresponding to the monoclinic phase being insulating and the orthorhombic one being metallic with uniform distribution of site occupancies (nondisproportionated metal). Because of the great sensitivity of the critical boundary to $\Delta_{s}$, rather extended regions of the $(U, J)$ parameter space satisfy these conditions [basically corresponding to the area delimited by the orthorhombic (blue) boundary to the right and the monoclinic (red) one to the left, including the range $J=0.3-0.7$ and $U=1.5-2.0]$.

The calculated cRPA values are marked by a (yellow) diamond symbol on each panel of Fig. 4. They are located well within the metallic domain for the orthorhombic phase and just inside the insulating domain (rather close to the MIT boundary) for the monoclinic phase. These results demonstrate that cRPA is able to provide reasonable values of the effective screened interactions, which correctly account for the physical nature of each phase.

\section{DISCUSSION AND CONCLUSIONS}

In summary, the question we have addressed in this paper is that of the appropriate values of interaction parameters for rare-earth nickelates, when adopting a low-energy description of their electronic structure involving only the partially occupied $e_{g}$ states. We have calculated these effective lowenergy interaction parameters from first principles for $\mathrm{LuNiO}_{3}$, using the constrained random-phase approximation (cRPA). The obtained values confirm the strong reduction of the effective on-site $U$ by screening, down to $U \simeq 1.65 \mathrm{eV}$ in the orthorhombic phase $(U \simeq 1.83 \mathrm{eV}$ in the monoclinic phase), while the Hund's coupling $J$ remains sizable $(J \simeq$ $0.33,0.37 \mathrm{eV}$ in each phase, respectively).

The cRPA results also reveal the importance of the longrange intersite interactions, with a slow spatial decay $V_{1} / R$. $V_{1}$ is found to be of the order of $0.42-0.44 \mathrm{eV}$ so that these interactions must be included in a proper low-energy treatment. When treated at the level of a Hartree approximation, they lead to a further reduction of the effective parallel-spin local interaction $U_{\text {eff }}^{\sigma \sigma}=U-3 J-z_{\text {eff }} V_{1}$ (with $z_{\text {eff }}$ the effective Madelung connectivity), which is found to be small and negative. This is qualitatively consistent with the picture of a negative charge transfer insulator and validates the low-energy description advocated in earlier work $[13,14]$. Let us also note that the low-energy interaction is further renormalized by higher-order many-body effects not taken into account in the present work, as well as by dynamical screening due to phonon modes. A rough estimate of the latter effect in nickelates suggests that it is small, but a more detailed study is left for future work. We have constructed an appropriate low-energy model based on the cRPA effective interactions and solved this model in the DFT+DMFT framework. We found that the monoclinic structure falls within the bonddisproportionated-insulator region, while the orthorhombic structure is located deep in the uniform metallic state, in agreement with experimental observations. While our calculations take into account only electronic degrees of freedom, a full theory of the metal-insulator transition in nickelates should also take into account the coupling to the relevant lattice mode associated with the structural transition: this should be addressed in future work.

Besides the specific example of nickelates, our work can be put in the broader context of compounds with small or negative charge transfer leading to the possible formation of ligand holes. We have shown that it is possible to build an appropriate low-energy effective theory of such compounds, involving only electronic states near the Fermi level, provided the strong reduction of the low-energy effective interactions is properly taken into account. This provides a perspective on these materials which is complementary to the one in which ligand states are explicitly retained in the description [5-7]. Future work should document the general applicability of the present approach by considering other compounds with small or negative charge transfer.

\section{ACKNOWLEDGMENTS}

We are grateful to Manuel Bibes, Sara Catalano, Claude Ederer, Marta Gibert, Alexander Hampel, Marisa Medarde, Andrew J. Millis, Yusuke Nomura, Swarup Panda, Julien Ruppen, George Sawatzky, Hugo Strand, Alaska Subedi, JeanMarie Tarascon, Jérémie Teyssier, Jean-Marc Triscone, Dirk van der Marel, and Julien Varignon for numerous discussions about the physics of rare-earth nickelates. This work was supported by the European Research Council Grants No. ERC319286-"QMAC" (A.G.), No. ERC-278472-"MottMetals" (O.P., P.S.), and No. ERC-617196-"CorrelMat” (S.B., P.S.), and IDRIS/GENCI Orsay under Project No. 201601393. L.P. acknowledges computational resources provided by the Swedish National Infrastructure for Computing (SNIC) at the National Supercomputer Centre (NSC) and PDC Center for High Performance Computing. O.P. and A.G. acknowledge support from the Swiss National Science Foundation NCCR MARVEL and computing resources provided by the Swiss National Supercomputing Centre (CSCS) under Projects No. s575 and No. mr17. M.B. gratefully acknowledges financial support from the Helmholtz Association through the Hemholtz Postdoctoral Programme (Grant No. VH-PD-022). F.A. acknowledges financial support from the Swedish Research Council (VR).
[1] M. Imada, A. Fujimori, and Y. Tokura, Rev. Mod. Phys. 70, 1039 (1998).
[2] F. C. Zhang and T. M. Rice, Phys. Rev. B 37, 3759 (1988). 
[3] J. Zaanen, G. A. Sawatzky, and J. W. Allen, Phys. Rev. Lett. 55, 418 (1985).

[4] J. Varignon, M. N. Grisolia, J. Iñiguez, A. Barthélémy, and M. Bibes, Quant. Mat. 2, 21 (2017).

[5] T. Mizokawa, H. Namatame, A. Fujimori, K. Akeyama, H. Kondoh, H. Kuroda, and N. Kosugi, Phys. Rev. Lett. 67, 1638 (1991).

[6] T. Mizokawa, A. Fujimori, H. Namatame, K. Akeyama, and N. Kosugi, Phys. Rev. B 49, 7193 (1994).

[7] T. Mizokawa, D. I. Khomskii, and G. A. Sawatzky, Phys. Rev. B 61, 11263 (2000).

[8] S. Johnston, A. Mukherjee, I. Elfimov, M. Berciu, and G. A. Sawatzky, Phys. Rev. Lett. 112, 106404 (2014).

[9] H. U. R. Strand, Phys. Rev. B 90, 155108 (2014).

[10] A. Demourgues, F. Weill, B. Darriet, A. Wattiaux, J. Grenier, P. Gravereau, and M. Pouchard, J. Solid State Chem. 106, 330 (1993).

[11] V. Bisogni, S. Catalano, R. J. Green, M. Gibert, R. Scherwitzl, Y. Huang, V. N. Strocov, P. Zubko, S. Balandeh, J.-M. Triscone, G. Sawatzky, and T. Schmitt, Nat. Commun. 7, 13017 (2016).

[12] H. Park, A. J. Millis, and C. A. Marianetti, Phys. Rev. Lett. 109, 156402 (2012).

[13] A. Subedi, O. E. Peil, and A. Georges, Phys. Rev. B 91, 075128 (2015).

[14] I. I. Mazin, D. I. Khomskii, R. Lengsdorf, J. A. Alonso, W. G. Marshall, R. M. Ibberson, A. Podlesnyak, M. J. Martínez-Lope, and M. M. Abd-Elmeguid, Phys. Rev. Lett. 98, 176406 (2007).

[15] A. Georges, G. Kotliar, W. Krauth, and M. J. Rozenberg, Rev. Mod. Phys. 68, 13 (1996).

[16] J. Ruppen, J. Teyssier, O. E. Peil, S. Catalano, M. Gibert, J. Mravlje, J.-M. Triscone, A. Georges, and D. van der Marel, Phys. Rev. B 92, 155145 (2015).

[17] J. Ruppen, J. Teyssier, I. Ardizzone, O. E. Peil, S. Catalano, M. Gibert, J.-M. Triscone, A. Georges, and D. van der Marel, Phys. Rev. B 96, 045120 (2017).

[18] P. G. J. van Dongen, Phys. Rev. B 49, 7904 (1994).

[19] C. M. Varma, Phys. Rev. Lett. 75, 898 (1995).

[20] J. van den Brink, M. B. J. Meinders, J. Lorenzana, R. Eder, and G. A. Sawatzky, Phys. Rev. Lett. 75, 4658 (1995).

[21] T. Watanabe, H. Yokoyama, Y. Tanaka, and J.-i. Inoue, J. Phys. Chem. Solids 69, 3372 (2008).

[22] M. Aichhorn, H. G. Evertz, W. von der Linden, and M. Potthoff, Phys. Rev. B 70, 235107 (2004).

[23] A. Amaricci, A. Camjayi, K. Haule, G. Kotliar, D. Tanasković, and V. Dobrosavljević, Phys. Rev. B 82, 155102 (2010).

[24] T. Ayral, S. Biermann, and P. Werner, Phys. Rev. B 87, 125149 (2013).

[25] C. Février, S. Fratini, and A. Ralko, Phys. Rev. B 91, 245111 (2015).

[26] H. Terletska, T. Chen, and E. Gull, Phys. Rev. B 95, 115149 (2017).

[27] F. Aryasetiawan, M. Imada, A. Georges, G. Kotliar, S. Biermann, and A. I. Lichtenstein, Phys. Rev. B 70, 195104 (2004).

[28] T. Miyake and F. Aryasetiawan, Phys. Rev. B 77, 085122 (2008).

[29] L. Vaugier, H. Jiang, and S. Biermann, Phys. Rev. B 86, 165105 (2012).

[30] B.-C. Shih, Y. Zhang, W. Zhang, and P. Zhang, Phys. Rev. B 85, 045132 (2012).

[31] B. Amadon, T. Applencourt, and F. Bruneval, Phys. Rev. B 89, 125110 (2014).
[32] A. van Roekeghem, L. Vaugier, H. Jiang, and S. Biermann, Phys. Rev. B 94, 125147 (2016).

[33] S. K. Panda, H. Jiang, and S. Biermann, Phys. Rev. B 96, 045137 (2017).

[34] P. Seth, P. Hansmann, A. van Roekeghem, L. Vaugier, and S. Biermann, Phys. Rev. Lett. 119, 056401 (2017).

[35] J. A. Alonso, M. J. Martínez-Lope, M. T. Casais, J. L. GarcíaMuñoz, and M. T. Fernández-Díaz, Phys. Rev. B 61, 1756 (2000).

[36] C. Friedrich, S. Blügel, and A. Schindlmayr, Phys. Rev. B 81, $125102(2010)$

[37] S. Blügel and G. Bihlmayer, in Computational Nanoscience: Do It Yourself! NIC Series Vol. 31, edited by J. Grotendorst, S. Blügel, and D. Marx (John von Neumann Institute for Computing, Jülich, 2006), p. 85; http://www.flapw.de.

[38] A. A. Mostofi, J. R. Yates, Y.-S. Lee, I. Souza, D. Vanderbilt, and N. Marzari, Comput. Phys. Commun. 178, 685 (2008).

[39] P. Hansmann, L. Vaugier, H. Jiang, and S. Biermann, J. Phys.: Condens. Matter 25, 094005 (2013).

[40] P. Hansmann, T. Ayral, L. Vaugier, P. Werner, and S. Biermann, Phys. Rev. Lett. 110, 166401 (2013).

[41] P. Hansmann, T. Ayral, A. Tejeda, and S. Biermann, Sci. Rep. 6, 19728 (2016).

[42] T. Miyake, F. Aryasetiawan, and M. Imada, Phys. Rev. B 80, 155134 (2009).

[43] E. Şaşığlu, C. Friedrich, and S. Blügel, Phys. Rev. B 83, 121101 (2011).

[44] We mention in passing that we have developed an application of the TRIQS/DFTTOOLS package [46,71-73] which provides an interface to the FLEUR electronic structure code .

[45] C. Friedrich, A. Schindlmayr, S. Blügel, and T. Kotani, Phys. Rev. B 74, 045104 (2006).

[46] G. Michalicek, M. Betzinger, C. Friedrich, and S. Blügel, Comput. Phys. Commun. 184, 2670 (2013).

[47] M. Casula, P. Werner, L. Vaugier, F. Aryasetiawan, T. Miyake, A. J. Millis, and S. Biermann, Phys. Rev. Lett. 109, 126408 (2012).

[48] P. Werner, M. Casula, T. Miyake, F. Aryasetiawan, A. J. Millis, and S. Biermann, Nat. Phys. 8, 331 (2012).

[49] A. van Roekeghem, T. Ayral, J. M. Tomczak, M. Casula, N. Xu, H. Ding, M. Ferrero, O. Parcollet, H. Jiang, and S. Biermann, Phys. Rev. Lett. 113, 266403 (2014).

[50] T. Miyake, C. Martins, R. Sakuma, and F. Aryasetiawan, Phys. Rev. B 87, 115110 (2013).

[51] J. M. Tomczak, M. Casula, T. Miyake, and S. Biermann, Phys. Rev. B 90, 165138 (2014).

[52] S. Biermann, F. Aryasetiawan, and A. Georges, Phys. Rev. Lett. 90, 086402 (2003).

[53] K. J. Kapcia, S. Robaszkiewicz, M. Capone, and A. Amaricci, Phys. Rev. B 95, 125112 (2017).

[54] J. M. Tomczak, M. Casula, T. Miyake, F. Aryasetiawan, and S. Biermann, Europhys. Lett. 100, 67001 (2012).

[55] L. Boehnke, F. Nilsson, F. Aryasetiawan, and P. Werner, Phys. Rev. B 94, 201106 (2016).

[56] H. Shinaoka, M. Troyer, and P. Werner, Phys. Rev. B 91, 245156 (2015).

[57] F. Aryasetiawan, R. Sakuma, and K. Karlsson, Phys. Rev. B 85, 035106 (2012).

[58] M. Kinza and C. Honerkamp, Phys. Rev. B 92, 045113 (2015). 
[59] M. Schüler, M. Rösner, T. O. Wehling, A. I. Lichtenstein, and M. I. Katsnelson, Phys. Rev. Lett. 111, 036601 (2013).

[60] J. Merino and R. H. McKenzie, Phys. Rev. Lett. 87, 237002 (2001).

[61] T. Ayral, P. Werner, and S. Biermann, Phys. Rev. Lett. 109, 226401 (2012).

[62] L. Huang, T. Ayral, S. Biermann, and P. Werner, Phys. Rev. B 90, 195114 (2014).

[63] T. Yoshida and C. Hotta, Phys. Rev. B 90, 245115 (2014).

[64] E. G. C. P. van Loon, M. I. Katsnelson, and M. Lemeshko, Phys. Rev. B 92, 081106 (2015).

[65] T. Ayral, S. Biermann, and P. Werner, Phys. Rev. B 94, 239906(E) (2016).

[66] E. G. C. P. van Loon, M. Schüler, M. I. Katsnelson, and T. O. Wehling, Phys. Rev. B 94, 165141 (2016).

[67] T. Ayral, S. Biermann, P. Werner, and L. Boehnke, Phys. Rev. B 95, 245130 (2017).

[68] P. Blaha, K. Schwarz, G. K. H. Madsen, D. Kvasnicka, and J. Luitz, WIEN2K, An Augmented Plane Wave + Local
Orbitals Program for Calculating Crystal Properties (Karlheinz Schwarz, Technische Universität Wien, Austria, 2001).

[69] J. P. Perdew, K. Burke, and M. Ernzerhof, Phys. Rev. Lett. 77, 3865 (1996).

[70] B. Amadon, F. Lechermann, A. Georges, F. Jollet, T. O. Wehling, and A. I. Lichtenstein, Phys. Rev. B 77, 205112 (2008).

[71] M. Aichhorn, L. Pourovskii, V. Vildosola, M. Ferrero, O. Parcollet, T. Miyake, A. Georges, and S. Biermann, Phys. Rev. B 80, 085101 (2009).

[72] M. Aichhorn, L. Pourovskii, P. Seth, V. Vildosola, M. Zingl, O. E. Peil, X. Deng, J. Mravlje, G. J. Kraberger, C. Martins, M. Ferrero, and O. Parcollet, Comput. Phys. Commun. 204, 200 (2016).

[73] O. Parcollet, M. Ferrero, T. Ayral, H. Hafermann, I. Krivenko, L. Messio, and P. Seth, Comp. Phys. Commun. 196, 398 (2015).

[74] P. Seth, I. Krivenko, M. Ferrero, and O. Parcollet, Comput. Phys. Commun. 200, 274 (2016).

[75] V. I. Anisimov, F. Aryasetiawan, and A. I. Lichtenstein, J. Phys.: Condens. Matter 9, 767 (1997). 